\title{
Numerical and Experiential Study of Motion Control Using Pressure Feedback
}

\author{
M. B. Kjelland and M. R. Hansen \\ Department of Engineering, University of Agder, Grimstad, Norway \\ E-mail: magnus.b.kjelland@uia.no, michael.r.hansen@uia.no
}

\begin{abstract}
This paper is concerned with the inherent oscillatory nature of pressure compensated motion control of a hydraulic cylinder subjected to a negative load and suspended by means of an overcenter valve. A pressure feedback scheme that indirectly eliminates the oscillations is investigated. The indirect control scheme utilizes pressure feedback to electronically compensate the metering-out allowing for the removal of the compensator and, subsequently, elimination of the oscillations. The suggested electronic compensation scheme is implemented and examined in a single degree-of-freedom test rig actuated by means of a double acting hydraulic cylinder. The control scheme is compared with other control schemes and the importance of measurement filtering and controller cycle time are investigated.
\end{abstract}

Keywords: Overcenter valve, pressure feedback, negative load, experimental verification

\section{Introduction}

The use of overcenter valves or counterbalance valves are widely used in hydraulics on such applications as cranes, telehandlers and winches as an integrated part in the actuator control. They are distributed to each actuator (degree of freedom) depending on the type of external loading. They a multi-functional and, normally, they serve at least the following functions:

- leak tight load holding

- shock absorption

- cavitation protection at load lowering

- load holding at pipe burst

- no drop before lift

It is, however, well known that they tend to introduce instability in a system, especially when the flow supply is pressure compensated. This is the case when the directional control valve that supplies flow to the actuator suspended by the overcenter valve is equipped with an internal compensator in series with the main spool; a so-called flow control valve. As the latter is frequently asked for by system manufacturers a major problem in present day hydraulics is to design pressure compensated systems containing overcenter valves that offer stable load lowering performance without unnecessarily compromising system efficiency and response.

Naturally, this problem has attracted a lot of attention with emphasis on modeling and parameter variation [1..16] revealing a number of common stabilizing characteristics. They include increased volume between directional valve and actuator, reduced pilot area ratio as well as reduced pay and inertia load, whereas the influence by a number of other design variables remain less obvious.

As pointed out in [7] the operation of the over-center valve is application sensitive, greatly increasing the complexity of choosing/designing it. Adding to the difficulties is the fact that hard to control quantities such as friction and hysteresis in the overcenter valve also are sources of instability [8]. Finally, it should be noted that stability only is one of several performance parameters for an over-center valve system. The different functionalities constitutes a set of design constraints whereas power consumption, stability, response time, load dependency, manufacturability and costs represents performance criteria.

The popularity of the pressure compensation is mainly due to basic functionality: it allows several actuators to be driven simultaneously with minimal influence between the different active circuits. However, it does also provide an important advantage in closed loop motion control because the valve flow is a well-defined function of the control signal removing the disturbing influence from fluctuating loads.

Therefore, this paper is concerned with the removal of the oscillatory nature of an overcenter valve system taking into account that the above mentioned design criteria cannot be ignored. In [16] an alternative approach that simply moves the main throttling from the overcenter valve to the return orifice of the main directional control valve is introduced. In that case the overcenter valve is piloted open during lowering and the system is stable, however, it is limited with respect to the load variations that can be handled. In [17] a pressure feedback scheme that has as target to maintain the high pass filtered pressure gradient equal to zero is adopted with a view to reduce oscillations while maintaining other 
performance criteria related to the motion control. It yields lead compensation with a markedly improved performance, however, it requires a valve with a bandwidth that is significantly higher than the mechanical-hydraulic system. Alternatively, if at least two pressure transducers are available it is possible to abandon the mechanical-hydraulic compensation and, subsequently, the source of the oscillations, by using the measured metering-out pressure drop to compensate variations in pressure drop via the spool position. In that case the overcenter valve is maintained with unaltered functionality and the ability to share flow between actuators and reject load disturbances is maintained. The extra use of pressure transducers is easily motivated by the increasing level of instrumentation and computational power in hydraulically actuated machinery today.

However, the experimental implementation is needed. The main contribution of this paper is the practical implementation of the proposed electronic pressure compensation scheme on a single degree-of-freedom test rig with nonlinear dynamic characteristics using typical commercially available valves.

\section{Considered system}

In fig. 1 the main components of a pressure compensated overcenter valve circuit are shown. They comprise a 4/3 way directional control valve (in lowering position) with a compensator in series that maintains the pressure drop across the main spool metering orifice approximately constant. Further, the circuit contains an actuator (here a double acting cylinder) and the overcenter valve. Finally, a shock valve and a suction valve (not shown) are typically inserted so that cavitation and pressure peaks caused by the dissipation of the kinetic energy of the payload inertia are avoided.

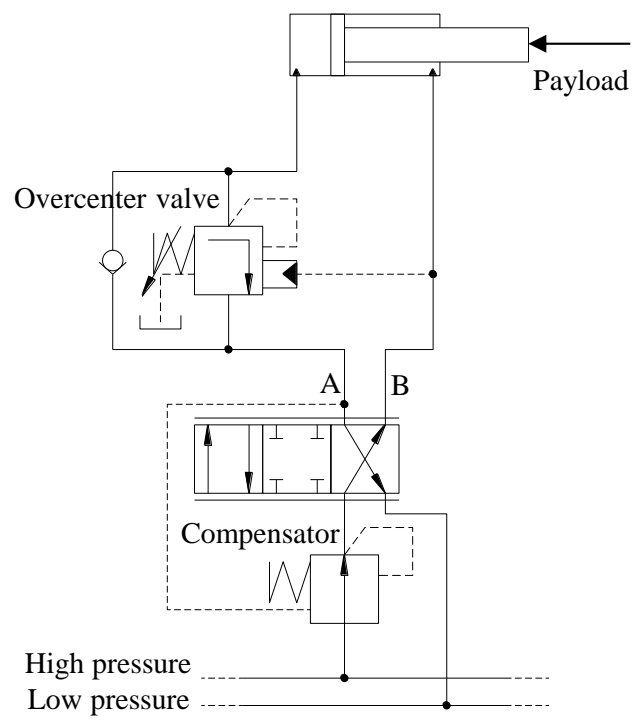

Figure 1: Single overcenter valve circuit with compensator.

In fig. 2 a simplified circuit is shown including the core components and parameters.

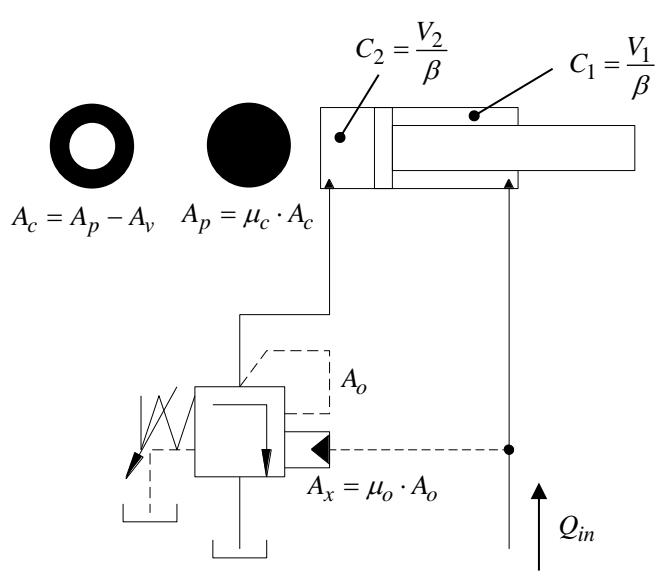

Figure 2: Simplified circuit.

Linearizing the governing equations for this system and formulating the transfer function between input signal and output velocity it is possible to utilize the Routh-Hurwitz stability criterion yielding:

$$
\frac{C_{1}}{C_{2}}>\frac{\mu_{o}}{\mu_{c}} \cdot \frac{K_{q o}}{K_{q o}+K_{q p}}
$$

In eq. (1) the flow gains of the overcenter valve with respect to valve opening pressure, $K_{q o}$, and valve pressure drop,

$K_{q p}$, appear together with the capacitances and the area ratios of the overcenter valve and the cylinder, respectively. Normally, $K_{q o} \gg K_{q p}$, hence, for normal operation and typical values for $\mu_{o}=2 . .8$ the stability criteria is impossible to satisfy in the major part of the cylinder stroke. The expression in (1) is based on hard parameters, i.e., damping, friction, and viscosity do not enter into these equations.

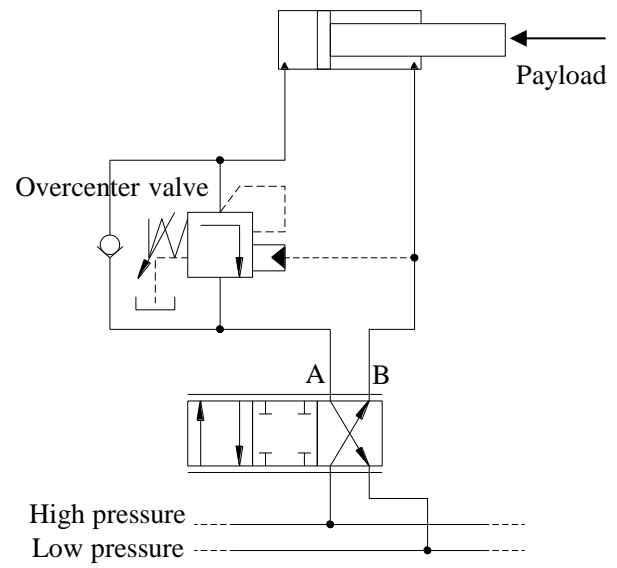

Figure 3: Single overcenter valve circuit without compensator.

The parameters that exhibit the strongest uncertainties are probably the capacitances via the influence of the bulk modulus of the fluid. However, it is clear that the basic overcenter valve circuit is prone to instability and oscillatory behavior. Removing the compensator, see fig. 3, eliminates the possible instability of the simplified circuit, in fact, the Routh-Hurwitz criterion now yields: 


$$
\mu_{c} \cdot C_{1}>0
$$

Which is always fulfilled for physical meaningful parameters. Another important stabilizing factor is that the non-compensated circuit will have more throttling across the return orifice yielding higher pressure levels and quite often a fully piloted open overcenter valve. In order to benefit from this it is, however, necessary to use a 4-port vented overcenter valve as indicated in figs. 1..3.

\section{Electronic pressure compensation}

The challenge with the circuit in fig. 3 is to facilitate flow sharing with several circuits active simultaneously and to reject load disturbances during motion control. The proposed electronic pressure compensation is based on an electro hydraulically actuated directional control valve with closed loop spool position control, see fig. 4.

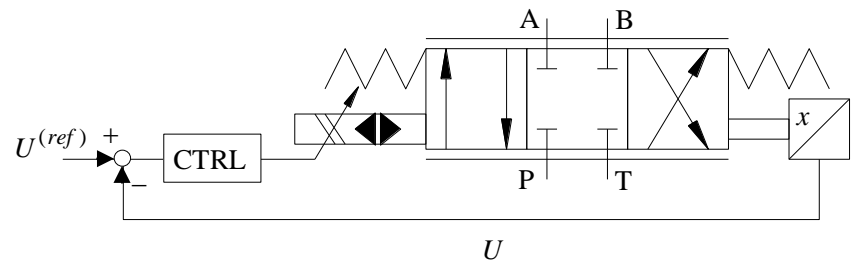

Figure 4: Inner spool position control loop.

For such a valve, we can assume that the steady state spool position will be a linear function of the input signal:

$$
x=K_{x U} \cdot U^{(r e f)}=K_{x U} \cdot U
$$

Also, the flow through the valve may be expressed using the orifice equation as:

$$
Q=C_{d} \cdot A_{d}(x) \cdot \sqrt{\frac{2}{\rho} \cdot \Delta p_{m o}}
$$

Combining eqs. (3) and (4) yields:

$$
Q=C_{v}(U) \cdot \sqrt{\Delta p_{m o}}
$$

where

$$
C_{v}(U)=C_{d} \cdot A_{d}\left(K_{x U} \cdot U\right) \cdot \sqrt{\frac{2}{\rho}}
$$

The variation of the discharge area is, in general, not a linear function, hence, some effort must be devoted to set up the inverse of eq. (6) yielding some functional relationship between the input signal, $U$, to the valve and the orifice parameter, $C_{v}$ :

$$
U=f\left(C_{v}\right)
$$

This functional relationship may be implemented in an electronic pressure compensation together with a continuous measurement of the pressure drop across the metering orifice to meet a flow reference, see fig. 5 .

Obviously, this is a purely open loop control of the valve flow, however, that corresponds to the classical pressure compensated system in fig. 1.

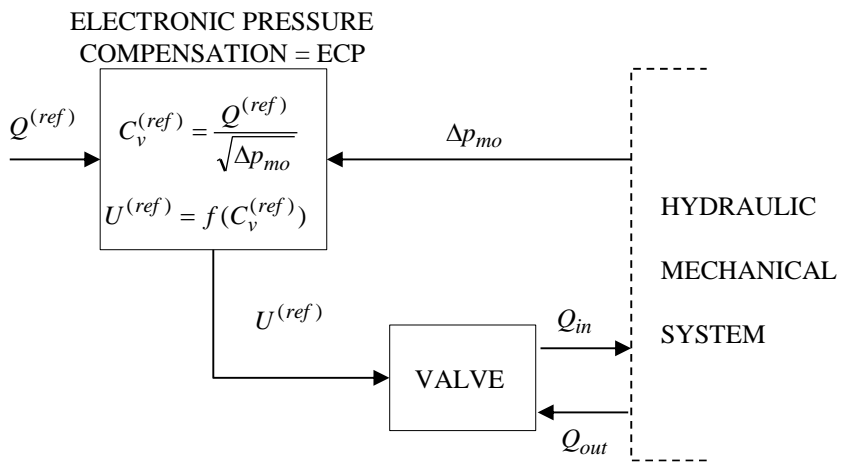

Figure 5: Basic components of electronic pressure compensation.

The electronic pressure compensation easily fits into a typical motion control scheme with a velocity feed forward term and a position feedback term, see fig. 6 .

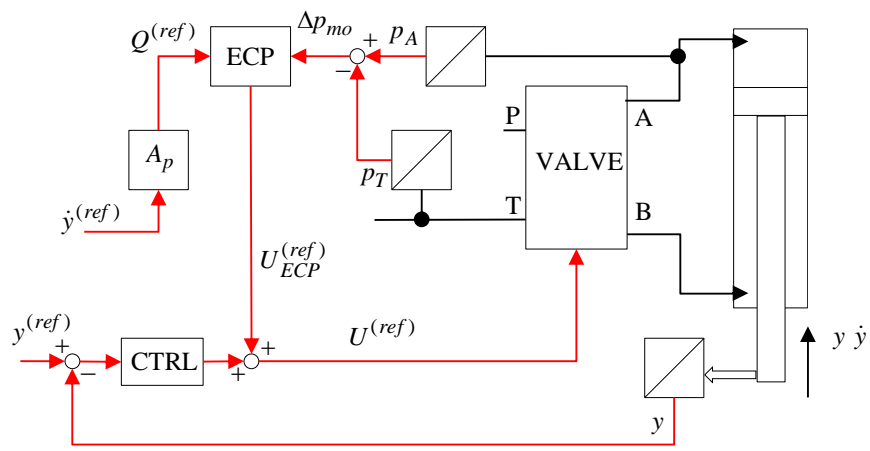

Figure 6: Electronic pressure compensation implemented in the feed forward path of a motion control scheme.

With position feedback from the actuator it is also possible to do the motion control without a variable feed forward term, i.e., without the electronic pressure compensation. This gives a simpler setup, however, it will be less efficient handling load disturbances or, in general, large load variations.

\section{Experimental setup}

In fig. 7 the experimental setup is shown in diagram form, and the mechanical dimensions are shown in fig. 8 .

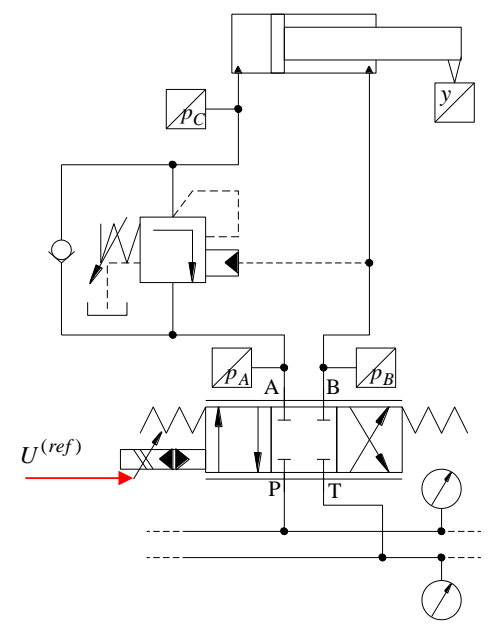

Figure 7: Hydraulic diagram of experimental setup. 
The directional control valve is part of a Sauer-Danfoss PVG32 valve group with several valve units both with and without pressure compensation. In fig. 7 is shown a valve with no compensator. The overcenter valve can be chosen from a range of Sun Hydraulics vented overcenter valves with pilot area ratio ranging from 1 to 5 .

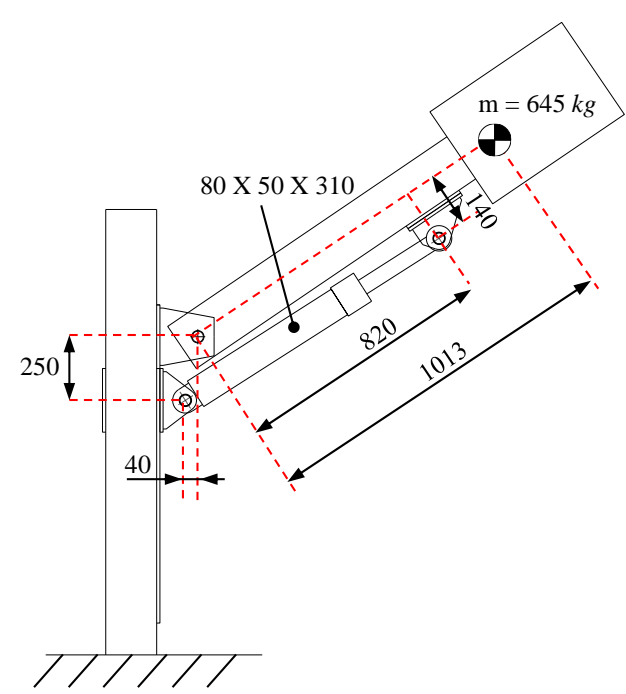

Figure 8: Mechanical system with all measurements in $\mathrm{mm}$.

The experimental setup is inserted in a ring line system with the fluid power being supplied by a pressure controlled HPU that maintains a pressure of 207 bar.

The electronic pressure compensation was only developed for the load lowering because this is where the system potentially becomes unstable or highly oscillatory. Also, the metering orifice is chosen as the return orifice A-T, for two reasons: it has a higher pressure drop during lowering thereby and the internal pressure drops are expected to be smaller in the outlet line of the valve as compared to the inlet thereby reducing the uncertainties associated with the actual pressure drop across the metering orifice.

For the main spool and the $24 \mathrm{~V}$ electrohydraulic actuation the curves in fig. 9 apply, as adopted from the PVG32 catalogue [18].
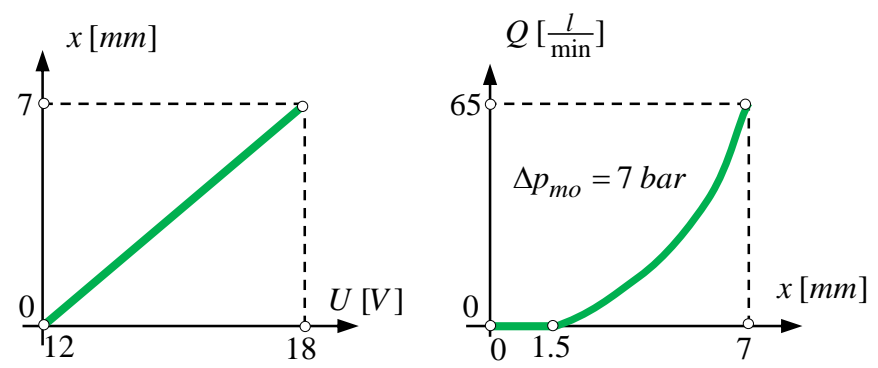

Figure 9: Spool travel vs. input signal (left) and volume flow vs. spool travel (right) for the main spool.

The mathematical expression for the flow as a function of the spool travel is expressed in eq. (8).

$$
\begin{aligned}
& Q(x)=0.327 \cdot x^{3}-2.236 \cdot x^{2}+10.716 \cdot x-12.319 \\
& {[Q]=\frac{l}{\min } \quad[x]=m m}
\end{aligned}
$$

From this a curve fit was developed to describe the variation of the input signal vs. the orifice parameter because this is what is ultimately needed in the electronic compensation, see fig. 5. In parallel, the same correlation was obtained experimentally by measuring the volume flow through the A-T orifice for four different pressure drops at different input signals. In fig. 10 the resulting curves are shown, and clearly there are some deviations around the smallest openings including a difference in actual dead band. The curve fit based on the measurements were used in the electronic compensation scheme since they easily produced better motion control performance than those derived from the catalogue. The entire valve characteristics were not mapped in this work since only the portion displayed in fig. 10 was used

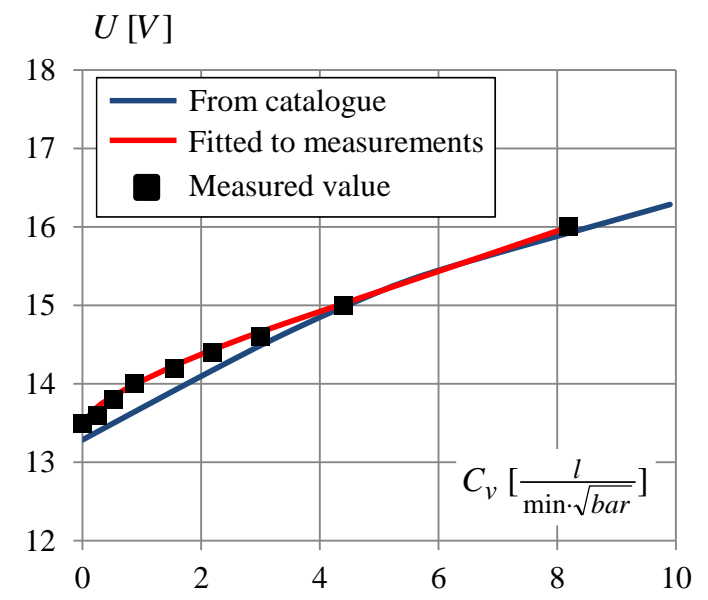

Figure 10: Input signal vs. orifice parameter as derived from catalogue data and from measurements.

The mathematical expression for the curve fitted to the measurements is given in eq. (9)

$$
\begin{aligned}
& U\left(C_{v}\right)=13.45+0.538 \cdot C_{v}^{0,699}+1.302 \cdot 10^{-6} \cdot C_{v}^{5.544} \\
& {[U]=V \quad\left[C_{v}\right]=\frac{l}{\min \cdot \sqrt{b a r}}}
\end{aligned}
$$

Continuous pressure measurements were only carried out for $p_{A}$ because the tank pressure outside the valve consistently was read to $p_{T}=0$ bar. The actual back pressure at the metering orifice, however, was estimated based on the current flow to be as high as $\Delta p_{\text {ret }}=1.5 \mathrm{bar}$. This was included in the electronic pressure compensation and had some correcting influence for situations with small $p_{A}$ values.

\section{Experimental results}

For the experimental investigations emphasis has been on lowering. In fig. 11 the inlet pressure, $p_{B}$, is plotted when lowering the arm by retracting the cylinder. In both cases an overcenter valve with pilot area ratio $\mu_{o}=3$ is used and the cylinder is retracted a distance $\Delta y=0.2 \mathrm{~m}$ in $\Delta t=5 \mathrm{~s}$. 

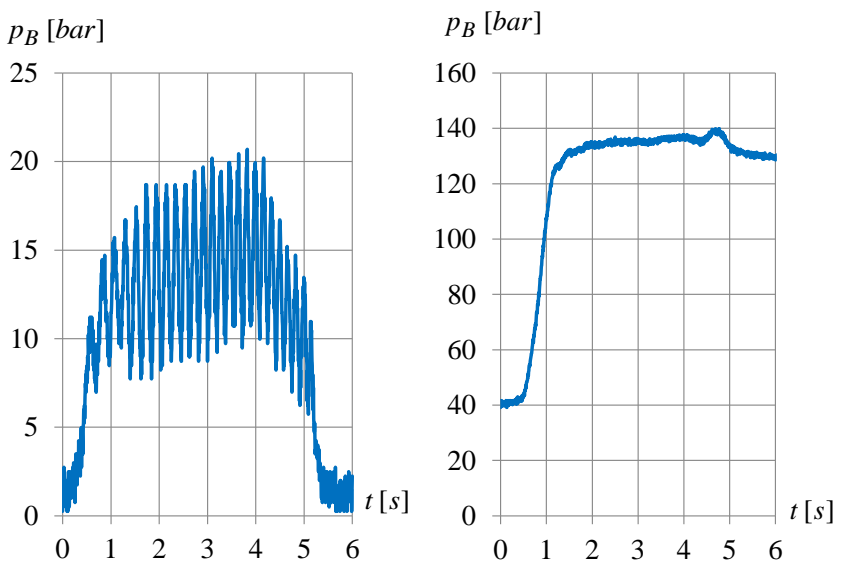

Figure 11: Inlet pressure, $p_{B}$, vs. time during cylinder retraction for a compensated (left) and a non-compensated (right) system.

It is apparent that removing the compensator eliminates the oscillations. In this case, the overcenter valve is simply piloted open yielding a very stable system.

Next, the control scheme in fig. 6 is implemented, however, in two versions: variable feed forward gain with continuous measurement of $\Delta p_{m o}$ and constant feed forward gain based on a fixed value of $\Delta p_{m o}$ which is estimated. The latter approach basically corresponds to tuning the feed forward gain to handle a range of pressure levels as good as possible. The pressure level is adjusted by means of the overcenter valve to three typical values and the reference motion is derived from a trapezoid shaped velocity profile that retracts the cylinder $\Delta y=0.3 m$ in $\Delta t=5 \mathrm{~s}$, see fig. 12 .
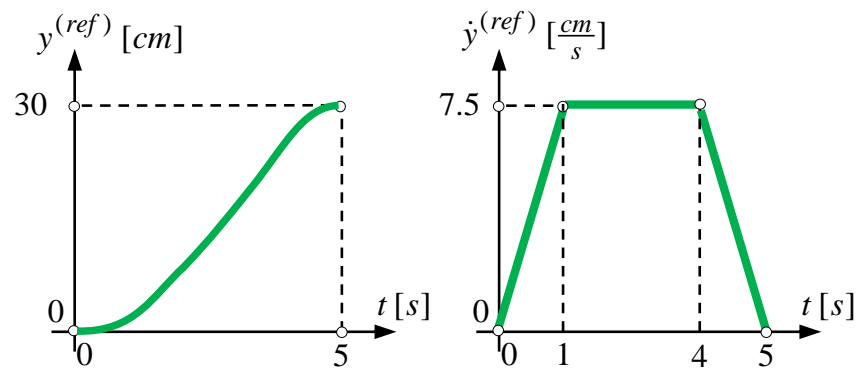

Figure 12: Reference position and velocity for cylinder.

In figs. 13 and 14 the resulting position error is shown for the variable and the constant feed forward gain. The errors are logged for three different load cases that are characterized by an average value for $p_{A}$ of 15,60 and 125 $b a r$, respectively. The back pressure is adjusted by means of the crack pressure of the overcenter valve while draining the pilot line.

The position error at the end of the $5 s$ travel is eventually removed by means of the position feedback control. As can be deduced from figs. 14 and 15 the constant feed forward gain was calibrated to yield the best possible result for a pressure drop of $\Delta p_{m o}=60 \mathrm{bar}$ across the metering orifice.

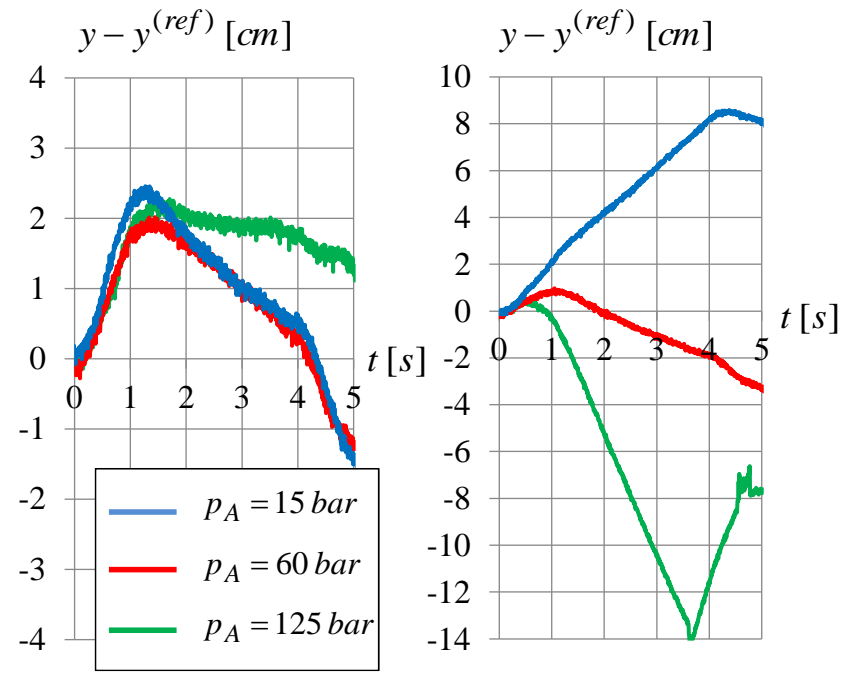

Figure 13: Position error for variable (left) and constant feed forward gain (right) without pressure feedback control.
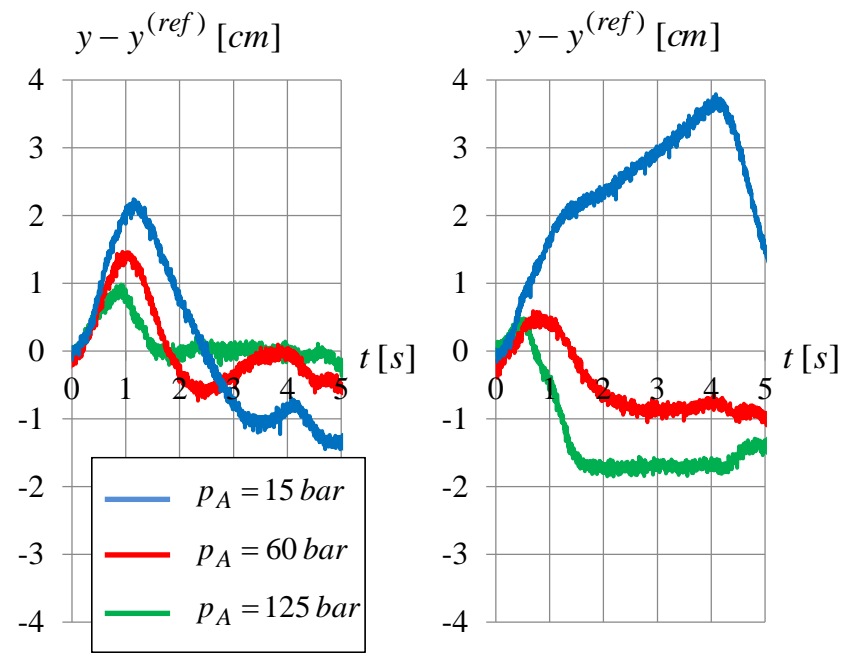

Figure 14: Position error for variable (left) and constant feed forward gain (right) with pressure feedback control.

In fig. 15 the input signal (subtracted the $12 \mathrm{~V}$ offset) and its contributions from the feed forward and the feedback path of the control scheme are shown for $p_{A}=125 \mathrm{bar}$.
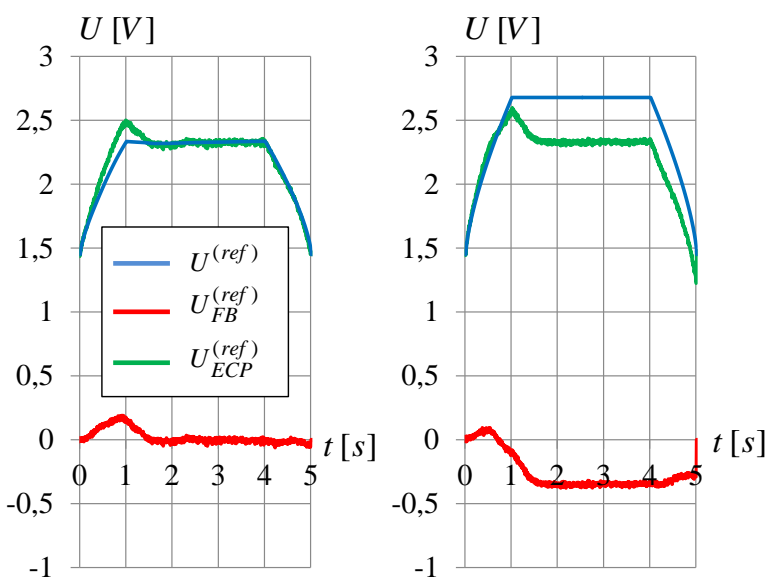

Figure 15: Valve input signal for variable (left) and constant feed forward gain (right). 
In general, the use of variable feed forward gain, i.e., electronic pressure compensation, reduced the demands on the closed loop controller. The price for this improved robustness is the introduction of pressure transducers and a somewhat more complex computation of the input signal. The main task may easily lie in the development of expressions like eqs. (8..9) that are needed in order for the proposed control scheme to work better than the constant feed forward.

In practice, the proposed control scheme may depend on the type of filtering of the pressure measurements as well as the cycle time of the controller. In the current implementation the filtering of the measurements is done via moving average and investigations clearly show that the method is quite robust towards the time span of the moving average. This reflects that it is not a rapidly varying parameters that is needed for the electronic pressure compensation but only a relatively slow varying steady state pressure level.

The influence of the cycle time is shown in fig. 16 where the position error is plotted vs. the cycle time.

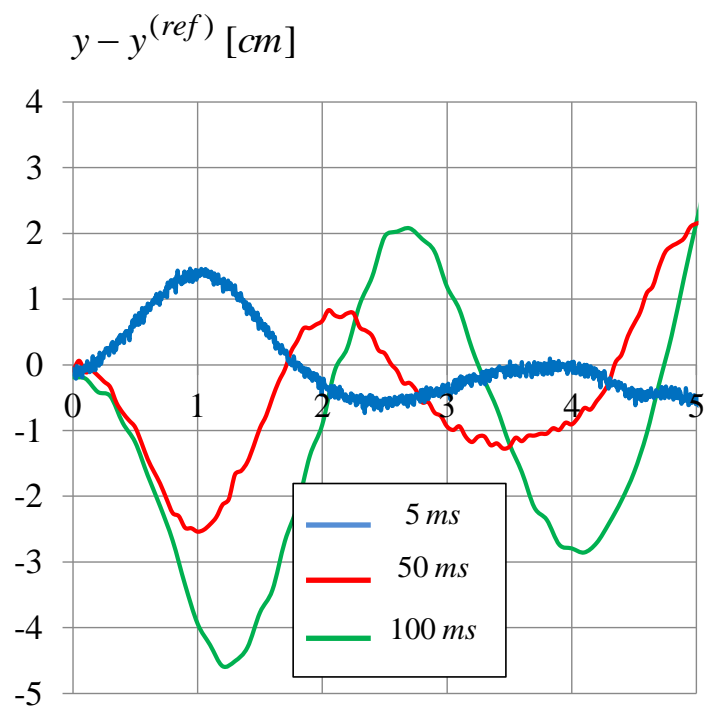

$t[s]$

Figure 16: Position error vs. cycle time.

It seems that at a cycle time around $50 \mathrm{~ms}$ the performance begins to deteriorate.

\section{Conclusions}

An electronic pressure compensation control scheme is put forward that allows the use of non-compensated directional control valves in closed loop motion control is presented and implemented in a practical system consisting of commercially available components. The control scheme measure the pressure drop across the metering-out orifice and continuously adjusts a feed forward gain that is computed based on a preprocessing of the valve characteristics. The main purpose of introducing the scheme is to reduce the inherently oscillatory nature of a pressure compensated valve in series with an overcenter valve. The experimental implementation reveals that abandoning the compensator eliminates the oscillatory nature of the hydraulic circuit. The electronic pressure compensation can be introduced in motion control via a velocity feed forward term but the valve catalogue data may not be adequate to represent the valve characteristics in a sufficiently precise manner. This seems to be most predominant at small openings of the valve. From the investigations conducted in this paper the proposed scheme gives a better performance than a pressure compensated circuit (difference in oscillation level) and a better performance than a non-compensated circuit with constant feed forward (less sensitive to load variations). In general, the proposed scheme seems to be a realistic alternative within motion control of hydraulic actuators.

\section{References}

[1] S Miyakawa. Stability of a Hydraulic Circuit With a Counterbalance-Valve, Bulletin of the JSME, 21(162), 1978.

[2] G. Overdiek, Dynamisches Verhalten von SenkbremsSperrventilen im Hydraulik-Hubwerk. Ölhydraulik und Pneumatik, 24(11), 1980 (in german).

[3] G Overdiek. Design and Characteristics of Hydraulic Winch Controls by Counterbalance Valves, European Conference on Hydrostatic Transmissions for Vehicle Applications, Aachen, 1981.

[4] T Persson, P Krus, and J O Palmberg. The Dynamic Properties of Over-Center Valves in Mobile Systems. Proc. of 2nd International Conference on Fluid Power Transmission and Control, Hangzhou, China, 1989.

[5] Y Ramli, P J Chapple, D G Tilley. An Application of computer aided design (CAD) in Hydraulic Systems Using Counterbalance Valves. Proc. of 4th International Conference on $C A D$ and $C G$, Wuhan, China, 1995.

[6] B Zähe. Stability of Load Holding Circuits with Counterbalance Valves. Proc. of 8th International Bath Fluid Power Workshop, Bath, UK, 1995.

[7] P J Chapple and D G Tilley. Evaluation Techniques For The Selection of Counterbalance Valves. Proc. of The Expo and Technical Conference for Electrohydraulic and Electropneumatic Motion Control Technology, Anaheim, USA, 1994

[8] H Handroos, J Halme and M Vilenius. Steady State and Dynamic Properties of Counterbalance Valves. Proc. of 3rd Scandinavian International Conference on Fluid Power, Linköping, Sweden, 1993.

[9] H. Zoebl. Senkbremsventile und Ventilkombinationen zur Steuerung der Bewegungsgeschwindigkeiten. Ölhydraulik und Pneumatik, 42(11-12), 1998 (in german). 
[10]F Weingarten. Aufbau Hydraulischer Zeitglieder und Ihr Einsatz im Signalzweig hydraulisch-mechanischer Regelungen. Dissertation Dr.-Ing., Fakultät für Maschinenwesen der Rheinisch-Westfälischen Technischen Hochschule, Aachen, 1983 (in german).

[11] T Persson, P Krus, and J O Palmberg. The Dynamic Properties of Over-Center Valves in Mobile Cranes. Proc. of 9th AFK Conference for Hydraulics and Pneumatics, Aachen, West Germany, 1990.

[12] M R Hansen, and T O Andersen. A Design Procedure for Actuator Control Systems Using Optimization. Proc. of The 7th Scandinavian International Conference on Fluid Power, Linköping, Sweden, 2001.

[13] T O Andersen, M R Hansen, P Pedersen, and F Conrad. The Influence of Flow Force Characteristics on the Performance of Over Centre Valves. Proc. of The 9th Scandinavian International Conference on Fluid Power, Linköping, Sweden, 2005.

[14] M R Hansen, and T O Andersen. Performance of Mobile Cranes Using Pressure Feedback. Proc. of 2001 Drives and Controls/Power Electronics Conference, London, UK, 2001.

[15] B Zähe, Allt under kontroll, Fluid Scandinavia, 2, 2009, (in swedish).

[16]P A Nordhammer, M K Bak and M R Hansen. A Method for Reliable Motion Control of Pressure Compensated Hydraulic Actuation With Counterbalance Valves. Proc. of 2012 ICCAS12 12th International Conference on Control, Automation, and Systems, Jeju, Korea, 2012.

[17] M R Hansen and $\mathrm{T} \quad \mathrm{O}$ Andersen. Controlling a negative loaded hydraulic cylinder using pressure feedback. Proc. of IASTED International Conference Modelling, Identification, and Control : Proceedings Innsbruck, Austria, 2010. 